\title{
Multinary Halogenido Bismuthates Beyond the Double Perovskite Motif
}

Natalie Dehnhardt, ${ }^{+}$Hayden Paneth, ${ }^{\ddagger}$ Nikolas Hecht, ${ }^{\S}$ Johanna Heine ${ }^{*}$,

† Department of Chemistry and Material Sciences Center, Philipps-Universität Marburg, Hans-Meerwein-Straße, 35043 Marburg, Germany.

¥ Department of Chemistry, Union College, Schenectady, New York 12308, USA.

$\S$ Department of Chemistry, Technische Universität Darmstadt, 64287 Darmstadt, Germany.

*Email: johanna.heine@chemie.uni-marburg.de 


\section{Crystallographic Details}

Single crystal X-ray determination was performed on a Bruker Quest D8 diffractometer with microfocus MoKa radiation and a Photon 100 (CMOS) detector $(\mathbf{1}, \mathbf{2}, \mathbf{3}, \mathbf{4}, \mathbf{5})$ or an a STOE IPDS$2 \mathrm{~T}$ diffractometer equipped with an imaging plate detector system using MoKa radiation with graphite monochromatization (6). All measurements were performed at $100 \mathrm{~K}$.

Table S1: Crystallographic data for $\left(\mathrm{PBz}_{4}\right)_{3} \mathrm{Bi}_{3} \mathrm{Br}_{12}$ (1), CCDC 1964160.

\begin{tabular}{|c|c|}
\hline Empirical formula & $\mathrm{C}_{84} \mathrm{H}_{84} \mathrm{Bi}_{3} \mathrm{Br}_{12} \mathrm{P}_{3}$ \\
\hline Formula weight & 2772.28 \\
\hline Crystal system & monoclinic \\
\hline Space group & $P 2_{1} / c$ \\
\hline$a / \AA$ & $12.3041(4)$ \\
\hline$b / \AA$ & $32.8540(12)$ \\
\hline$c / \AA$ & $22.3547(8)$ \\
\hline$\beta /{ }^{\circ}$ & $102.4080(10)$ \\
\hline Volume $/ \AA^{3}$ & $8825.6(5)$ \\
\hline $\mathrm{Z}$ & 4 \\
\hline$\rho_{\text {calc }} \mathrm{g} / \mathrm{cm}^{3}$ & 2.086 \\
\hline$\mu / \mathrm{mm}^{-1}$ & 11.496 \\
\hline $\mathrm{F}(000)$ & 5208.0 \\
\hline Crystal size $/ \mathrm{mm}^{3}$ & $0.13 \times 0.065 \times 0.059$ \\
\hline Absorption correction $\left(\mathrm{T}_{\min } / \mathrm{T}_{\max }\right)$ & multi-scan $(0.0607 / 0.0915)$ \\
\hline $2 \Theta$ range for data collection $/^{\circ}$ & 4.16 to 50.608 \\
\hline Index ranges & $-14 \leq \mathrm{h} \leq 14,-39 \leq \mathrm{k} \leq 39,-25 \leq 1 \leq 26$ \\
\hline Reflections collected & 147837 \\
\hline Independent reflections & $16065\left[R_{\text {int }}=0.1158, R_{\text {sigma }}=0.0560\right]$ \\
\hline Data/restraints/parameters & $16065 / 0 / 919$ \\
\hline Goodness-of-fit on $\mathrm{F}^{2}$ & 1.018 \\
\hline Final $\mathrm{R}$ indexes $[\mathrm{I}>=2 \sigma(\mathrm{I})]$ & $\mathrm{R}_{1}=0.0342, \mathrm{wR}_{2}=0.0450$ \\
\hline Final R indexes [all data] & $\mathrm{R}_{1}=0.0625, \mathrm{wR}_{2}=0.0500$ \\
\hline Largest diff. peak/hole / e $\AA^{-3}$ & $1.17 /-0.88$ \\
\hline
\end{tabular}

Details of crystal structure refinement: All non-hydrogen atoms were refined anisotropically. Hydrogen atoms were assigned to idealized geometric positions and included in structure factors calculations. 


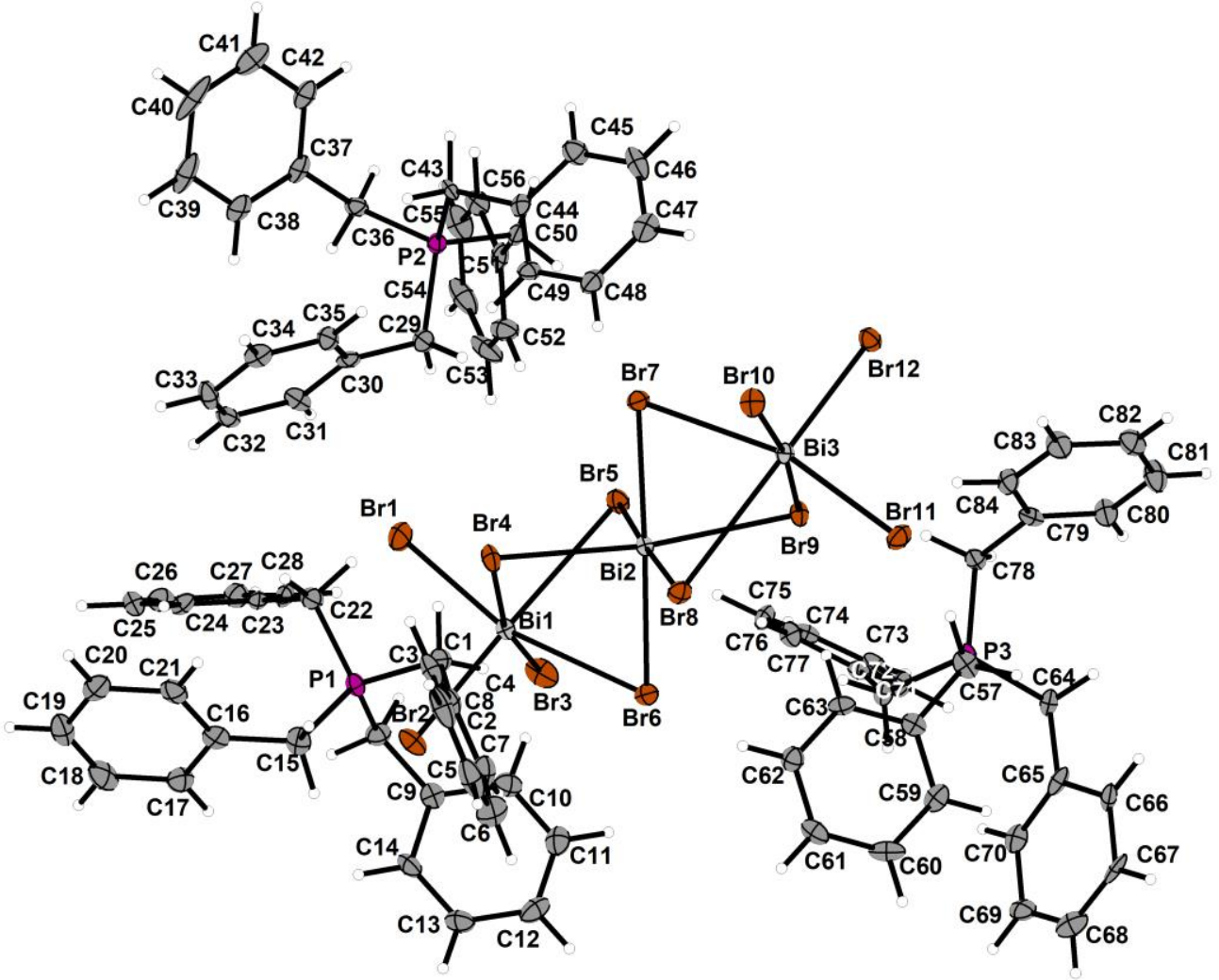

Figure S1: Asymmetric unit of 1, ellipsoids at 50\% probability. 
Table S2: Crystallographic data for $\left(\mathrm{PBz}_{4}\right)_{2}(\mathrm{MeCN})_{2} \mathrm{Cu}_{2} \mathrm{Bi}_{2} \mathrm{Br}_{10}(2), \mathrm{CCDC} 1964163$.

\begin{tabular}{|c|c|}
\hline Empirical formula & $\mathrm{C}_{60} \mathrm{H}_{62} \mathrm{Bi}_{2} \mathrm{Br}_{10} \mathrm{Cu}_{2} \mathrm{~N}_{2} \mathrm{P}_{2}$ \\
\hline Formula weight & 1108.60 \\
\hline Crystal system & triclinic \\
\hline Space group & $P-1$ \\
\hline$a / \AA$ & $11.0504(5)$ \\
\hline$b / \AA$ & $12.1103(5)$ \\
\hline$c / \AA$ & $13.8748(6)$ \\
\hline$\alpha /{ }^{\circ}$ & $81.6230(10)$ \\
\hline$\beta /{ }^{\circ}$ & $74.4050(10)$ \\
\hline$\gamma /{ }^{\circ}$ & $70.2340(10)$ \\
\hline Volume $/ \AA^{3}$ & $1679.98(13)$ \\
\hline $\mathrm{Z}$ & 1 \\
\hline$\rho_{\text {calc }} \mathrm{g} / \mathrm{cm}^{3}$ & 2.192 \\
\hline$\mu / \mathrm{mm}^{-1}$ & 11.878 \\
\hline $\mathrm{F}(000)$ & 1040.0 \\
\hline Crystal size $/ \mathrm{mm}^{3}$ & $0.328 \times 0.224 \times 0.188$ \\
\hline Absorption correction $\left(\mathrm{T}_{\min } / \mathrm{T}_{\max }\right)$ & multi-scan $(0.0076 / 0.0264)$ \\
\hline $2 \Theta$ range for data collection $/{ }^{\circ}$ & 4.446 to 50.656 \\
\hline Index ranges & $-13 \leq \mathrm{h} \leq 13,-14 \leq \mathrm{k} \leq 14,-16 \leq 1 \leq 16$ \\
\hline Reflections collected & 28264 \\
\hline Independent reflections & $6123\left[R_{\text {int }}=0.0538, R_{\text {sigma }}=0.0401\right]$ \\
\hline Data/restraints/parameters & $6123 / 0 / 353$ \\
\hline Goodness-of-fit on $\mathrm{F}^{2}$ & 1.081 \\
\hline Final $R$ indexes $[\mathrm{I}>=2 \sigma(\mathrm{I})]$ & $\mathrm{R}_{1}=0.0213, \mathrm{wR}_{2}=0.0510$ \\
\hline Final $\mathrm{R}$ indexes [all data] & $\mathrm{R}_{1}=0.0235, \mathrm{wR}_{2}=0.0519$ \\
\hline Largest diff. peak/hole / e $\AA^{-3}$ & $0.63 /-0.94$ \\
\hline
\end{tabular}

Details of crystal structure refinement: All non-hydrogen atoms were refined anisotropically. Hydrogen atoms were assigned to idealized geometric positions and included in structure factors calculations. 


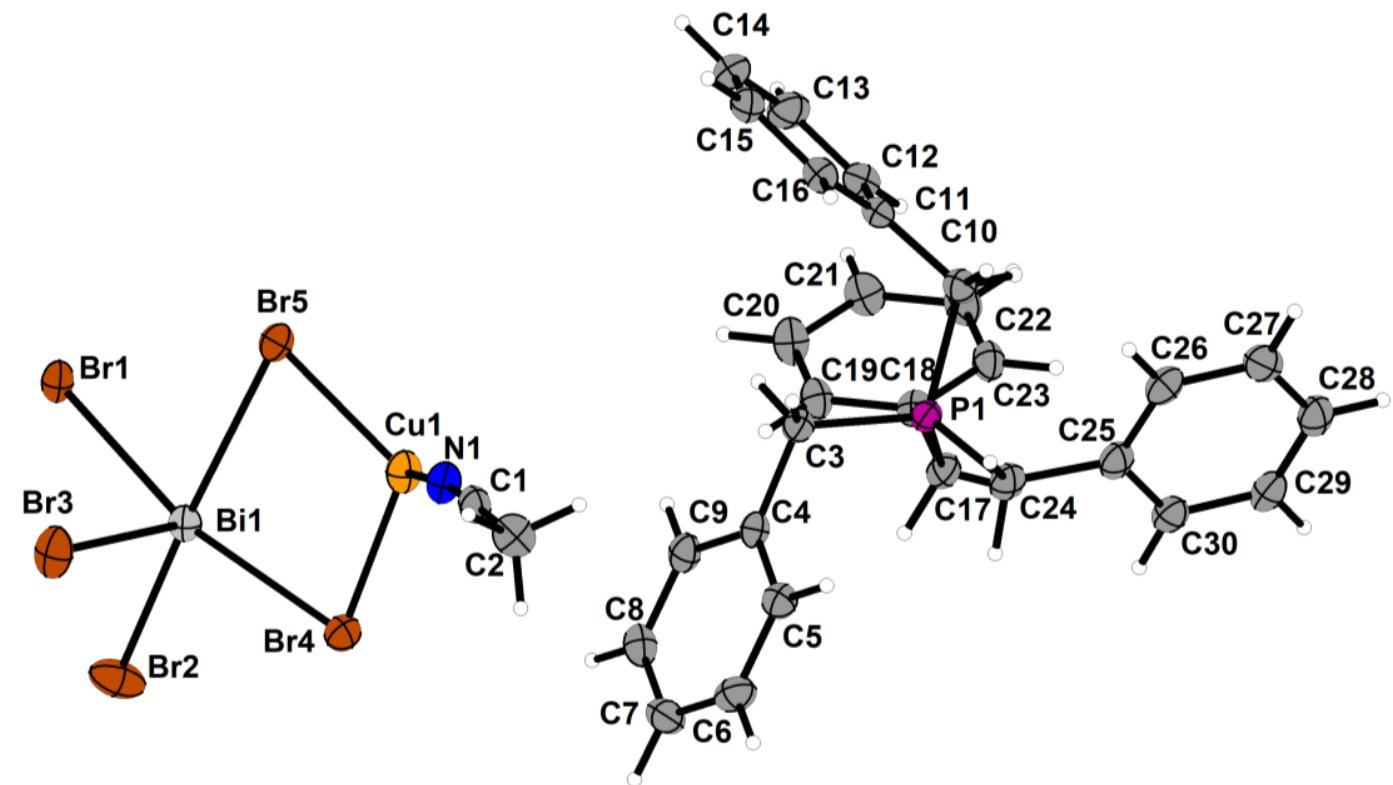

Figure S2: Asymmetric unit of 2, ellipsoids at 50\% probability. 
Table S3: Crystallographic data for $\left(\mathrm{PBz}_{4}\right) \mathrm{Bi}_{2} \mathrm{l}_{7}$ (3), CCDC 1964161.

\begin{tabular}{|c|c|}
\hline Empirical formula & $\mathrm{C}_{28} \mathrm{H}_{28} \mathrm{Bi}_{2} \mathrm{I}_{7} \mathrm{P}$ \\
\hline Formula weight & 1701.73 \\
\hline Crystal system & triclinic \\
\hline Space group & $P-1$ \\
\hline$a / \AA$ & $11.8654(6)$ \\
\hline$b / \AA$ & $12.9943(6)$ \\
\hline$c / \AA$ & $13.2031(6)$ \\
\hline$\alpha /{ }^{\circ}$ & $109.1820(10)$ \\
\hline$\beta /{ }^{\circ}$ & $91.763(2)$ \\
\hline$\gamma /{ }^{\circ}$ & $96.687(2)$ \\
\hline Volume $/ \AA^{3}$ & $1904.41(16)$ \\
\hline $\mathrm{Z}$ & 2 \\
\hline$\rho_{\text {calc }} g / \mathrm{cm}^{3}$ & 2.968 \\
\hline$\mu / \mathrm{mm}^{-1}$ & 14.961 \\
\hline $\mathrm{F}(000)$ & 1496.0 \\
\hline Crystal size $/ \mathrm{mm}^{3}$ & $0.419 \times 0.411 \times 0.2$ \\
\hline Absorption correction $\left(\mathrm{T}_{\min } / \mathrm{T}_{\max }\right)$ & numerical $(0.03282 / 0.17705)$ \\
\hline $2 \Theta$ range for data collection $/{ }^{\circ}$ & 4.486 to 50.638 \\
\hline Index ranges & $-14 \leq \mathrm{h} \leq 14,-15 \leq \mathrm{k} \leq 15,-15 \leq 1 \leq 15$ \\
\hline Reflections collected & 29850 \\
\hline Independent reflections & $6939\left[R_{\text {int }}=0.0617, R_{\text {sigma }}=0.0498\right]$ \\
\hline Data/restraints/parameters & $6939 / 42 / 343$ \\
\hline Goodness-of-fit on $\mathrm{F}^{2}$ & 1.102 \\
\hline Final $\mathrm{R}$ indexes $[\mathrm{I}>=2 \sigma(\mathrm{I})]$ & $\mathrm{R}_{1}=0.0422, \mathrm{wR}_{2}=0.1064$ \\
\hline Final $\mathrm{R}$ indexes [all data] & $\mathrm{R}_{1}=0.0447, \mathrm{wR}_{2}=0.1082$ \\
\hline Largest diff. peak/hole / e $\AA^{-3}$ & $2.21 /-5.91$ \\
\hline
\end{tabular}

Details of crystal structure refinement: All non-hydrogen atoms were refined anisotropically. Hydrogen atoms were assigned to idealized geometric positions and included in structure factors calculations. A number of ISOR restraints had to be used to obtain reasonable displacement parameters for some of the carbon atoms. The minimum residual density is comparatively high. We ascribe this to the fairly large crystal specimen that was chosen for measurement. 


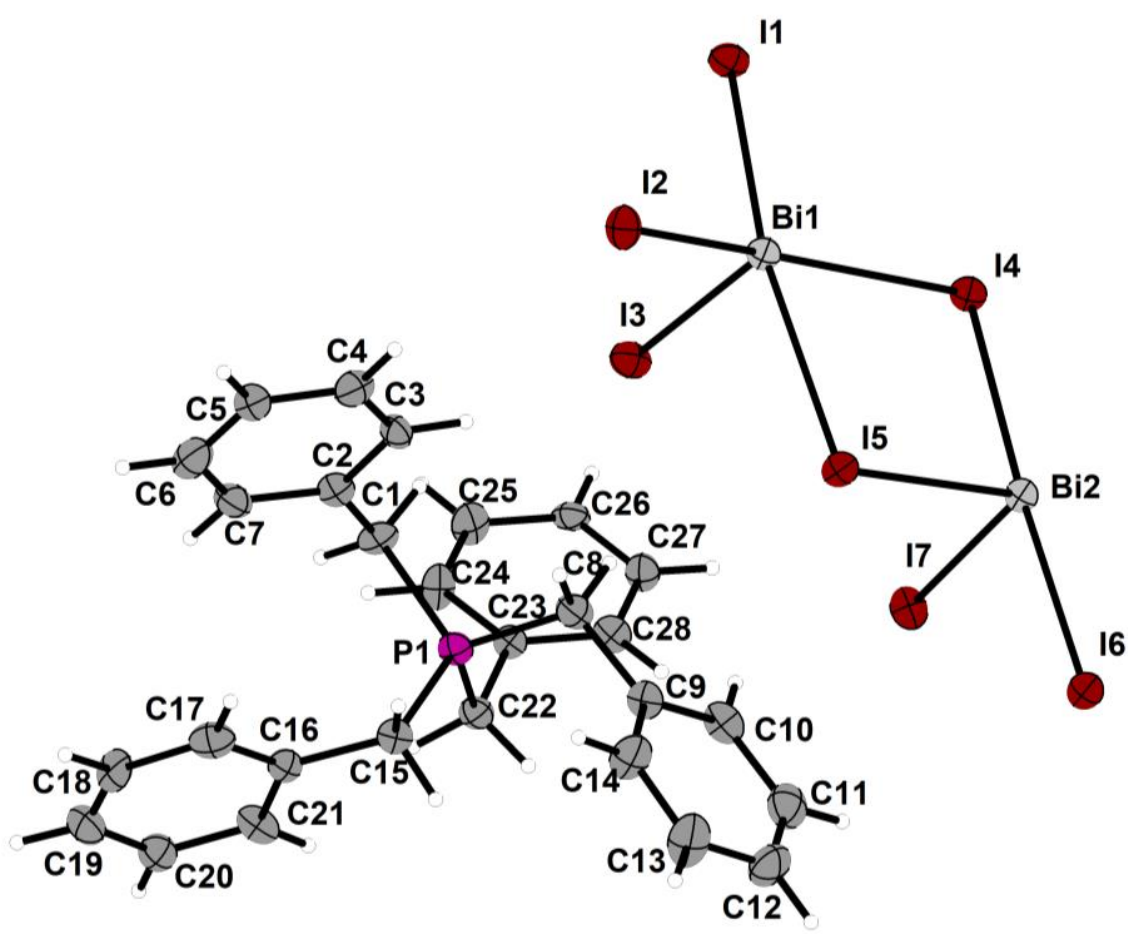

Figure S3: Asymmetric unit of 3, ellipsoids at 50\% probability. 
Table S4: Crystallographic data for $\left(\mathrm{PBz}_{4}\right)_{2}(\mathrm{MeCN})_{2} \mathrm{Cu}_{2} \mathrm{Bi}_{2} \mathrm{I}_{10}$ (4), CCDC 1964162.

$\begin{array}{ll}\text { Empirical formula } & \mathrm{C}_{60} \mathrm{H}_{62} \mathrm{Bi}_{2} \mathrm{Cu}_{2} \mathrm{I}_{10} \mathrm{~N}_{2} \mathrm{P}_{2} \\ \text { Formula weight } & 2687.09 \\ \text { Crystal system } & \text { triclinic } \\ \text { Space group } & \mathrm{P}-1 \\ a / \AA & 10.7763(5) \\ b / \AA & 12.5033(5) \\ c / \AA & 14.8812(7) \\ \alpha /{ }^{\circ} & 80.5280(10) \\ \beta /{ }^{\circ} & 76.0830(10) \\ \gamma /{ }^{\circ} & 70.2750(10) \\ \text { Volume/ } \AA^{3} & 1824.38(14) \\ \mathrm{Z} & 1 \\ \rho_{\text {calcg/cm }}{ }^{3} & 2.446 \\ \mu / \mathrm{mm}^{-1} & 9.694 \\ \mathrm{~F}(000) & 1220.0 \\ \text { Crystal size/mm } & \\ \text { Absorption correction }\left(\mathrm{T}_{\min } / \mathrm{T}_{\max }\right) & 0.28 \times 0.125 \times 0.073 \\ 2 \Theta \text { range for data collection }{ }^{\circ} & 4.278 \text { to } 50.624 \\ \text { Index ranges } & -12 \leq \mathrm{h} \leq 12,-15 \leq \mathrm{k} \leq 15,-17 \leq 1 \leq 17 \\ \text { Reflections collected } & 30276 \\ \text { Independent reflections } & 6639\left[\mathrm{R}_{\text {int }}=0.0294, \mathrm{R}_{\text {sigma }}=0.0230\right] \\ \text { Data/restraints/parameters } & 6639 / 0 / 353 \\ \text { Goodness-of-fit on } \mathrm{F}^{2} & 1.140 \\ \text { Final } \mathrm{R} \text { indexes }[\mathrm{I}>=2 \sigma(\mathrm{I})] & \mathrm{R}_{1}=0.0167, \mathrm{wR}_{2}=0.0369 \\ \text { Final R indexes [all data }] & \mathrm{R}_{1}=0.0192, \mathrm{wR}_{2}=0.0377 \\ \text { Largest diff. peak/hole / e } \AA^{-3} & 0.43 /-1.10 \\ & \end{array}$

Details of crystal structure refinement: All non-hydrogen atoms were refined anisotropically. Hydrogen atoms were assigned to idealized geometric positions and included in structure factors calculations. 


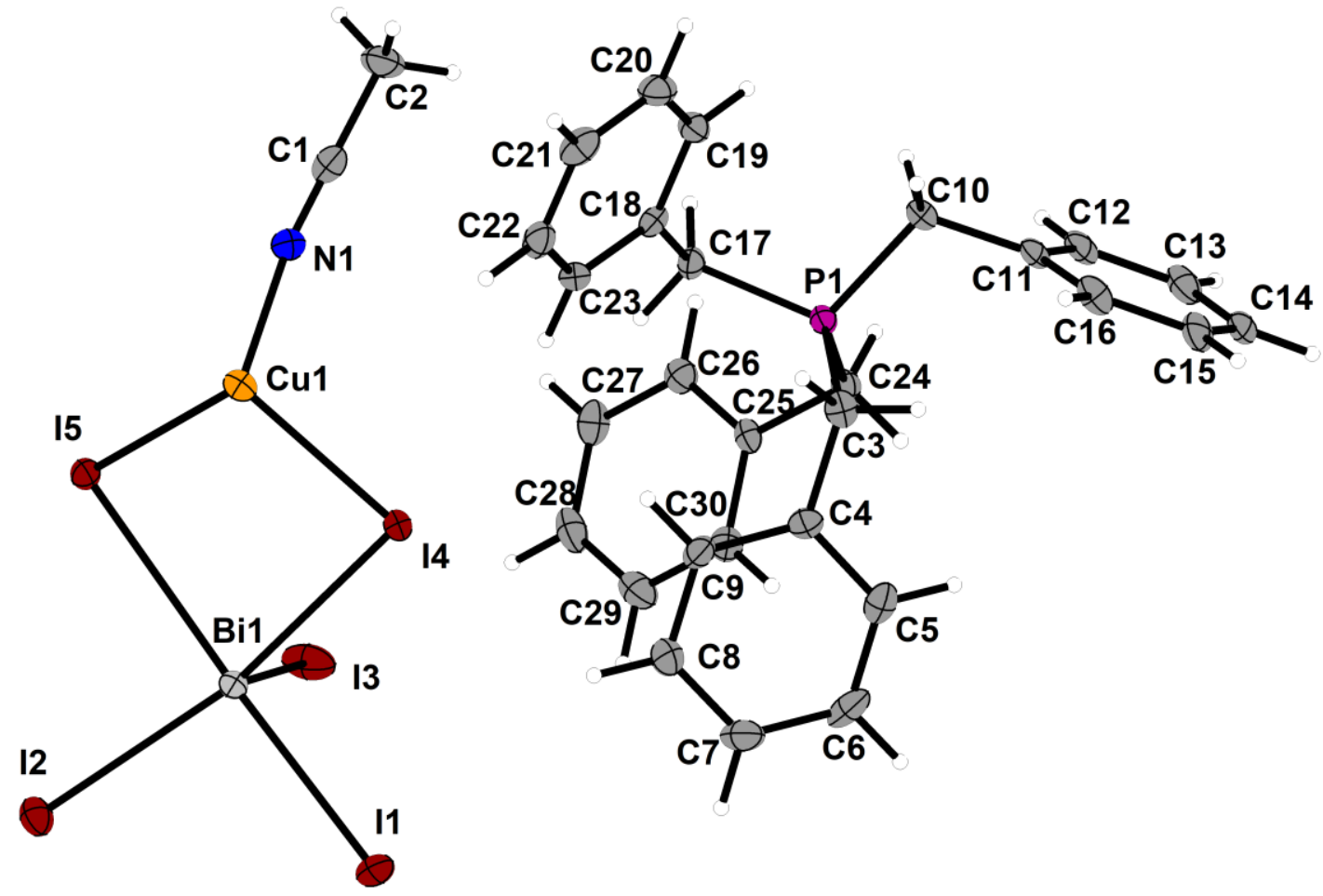

Figure S4: Asymmetric unit of 4, ellipsoids at 50\% probability. 
Table S5: Crystallographic data for $\left(\mathrm{PBz}_{4}\right)_{2} \mathrm{AgBi}_{2} \mathrm{I}_{9}(5), \mathrm{CCDC} 1964165$.

\begin{tabular}{|c|c|}
\hline Empirical formula & $\mathrm{C}_{56} \mathrm{H}_{56} \mathrm{AgBi}_{2} \mathrm{I}_{9} \mathrm{P}_{2}$ \\
\hline Formula weight & 2458.87 \\
\hline Crystal system & monoclinic \\
\hline Space group & $P 2_{1} / n$ \\
\hline$a / \AA$ & $24.2571(8)$ \\
\hline$b / \AA$ & $11.5623(4)$ \\
\hline$c / \AA$ & $25.4550(9)$ \\
\hline$\beta /{ }^{\circ}$ & $116.1780(10)$ \\
\hline Volume $/ \AA^{3}$ & $6407.0(4)$ \\
\hline $\mathrm{Z}$ & 4 \\
\hline$\rho_{\text {calc }} \mathrm{g} / \mathrm{cm}^{3}$ & 2.549 \\
\hline$\mu / \mathrm{mm}^{-1}$ & 10.203 \\
\hline $\mathrm{F}(000)$ & 4448.0 \\
\hline Crystal size $/ \mathrm{mm}^{3}$ & $0.082 \times 0.08 \times 0.068$ \\
\hline Absorption correction $\left(\mathrm{T}_{\min } / \mathrm{T}_{\max }\right)$ & multi-scan $(0.6424 / 0.7452)$ \\
\hline $2 \Theta$ range for data collection ${ }^{\circ}$ & 4.694 to 50.594 \\
\hline Index ranges & $-29 \leq \mathrm{h} \leq 29,-13 \leq \mathrm{k} \leq 13,-30 \leq 1 \leq 30$ \\
\hline Reflections collected & 105076 \\
\hline Independent reflections & $11644\left[\mathrm{R}_{\text {int }}=0.0474, \mathrm{R}_{\text {sigma }}=0.0226\right]$ \\
\hline Data/restraints/parameters & $11644 / 0 / 631$ \\
\hline Goodness-of-fit on $\mathrm{F}^{2}$ & 1.066 \\
\hline Final $\mathrm{R}$ indexes $[\mathrm{I}>=2 \sigma(\mathrm{I})]$ & $\mathrm{R}_{1}=0.0190, \mathrm{wR}_{2}=0.0320$ \\
\hline Final $\mathrm{R}$ indexes [all data] & $\mathrm{R}_{1}=0.0254, \mathrm{wR}_{2}=0.0331$ \\
\hline Largest diff. peak/hole / e $\AA^{-3}$ & $0.82 /-0.73$ \\
\hline
\end{tabular}

Details of crystal structure refinement: All non-hydrogen atoms were refined anisotropically. Hydrogen atoms were assigned to idealized geometric positions and included in structure factors calculations. 


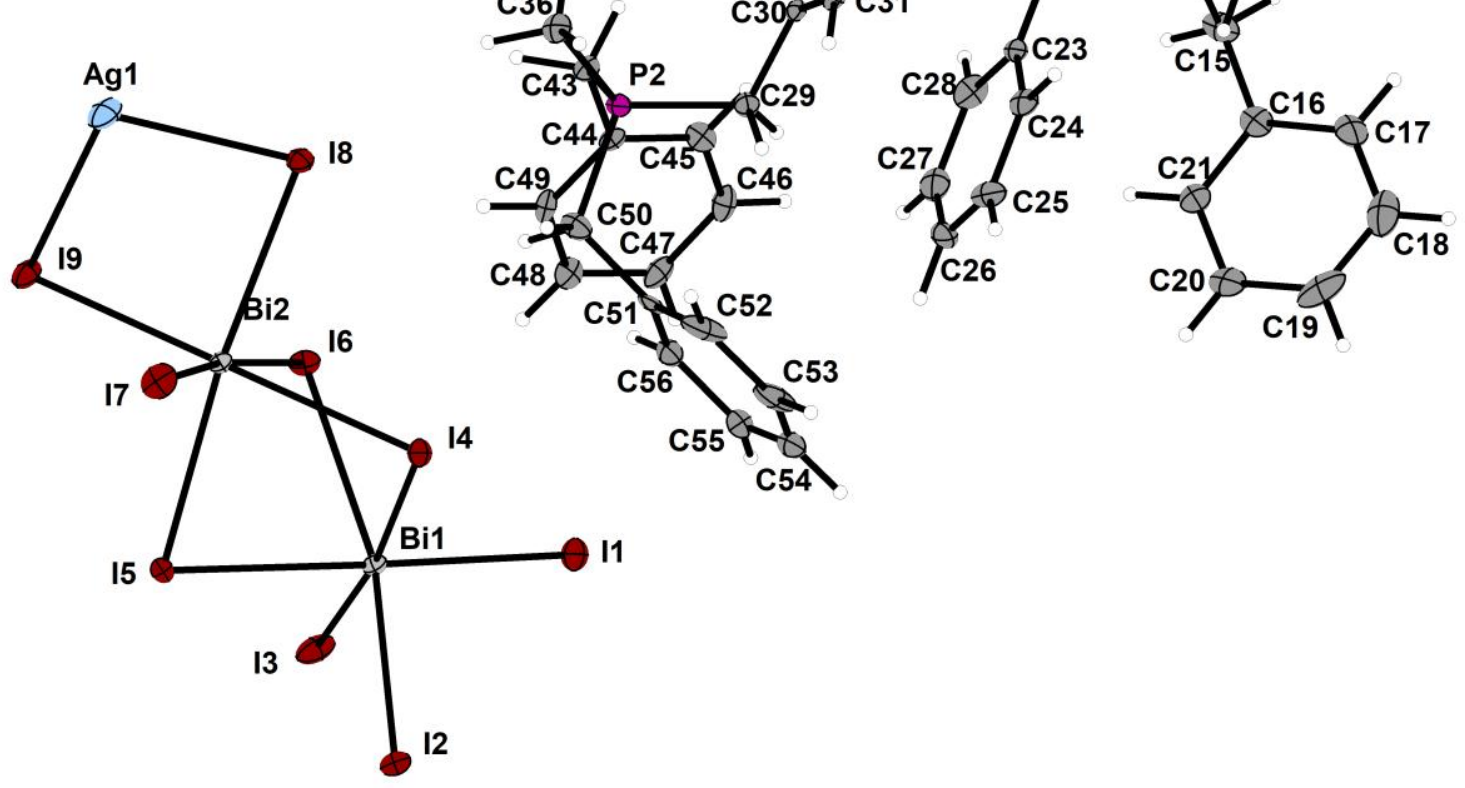

Figure S5: Asymmetric unit of 5, ellipsoids at 50\% probability. 
Table S6: Crystallographic data for $\left(\mathrm{PBz}_{4}\right)_{3} \mathrm{Bi}_{3} \mathrm{l}_{12} \cdot \mathrm{C}_{4} \mathrm{H}_{8} \mathrm{O}$ (6), CCDC 1964164.

\begin{tabular}{|c|c|}
\hline Empirical formula & $\mathrm{C}_{88} \mathrm{H}_{92} \mathrm{Bi}_{3} \mathrm{I}_{12} \mathrm{OP}_{3}$ \\
\hline Formula weight & 3408.26 \\
\hline Crystal system & monoclinic \\
\hline Space group & $P 2_{1} / c$ \\
\hline$a / \AA$ & $13.895(3)$ \\
\hline$b / \AA$ & $18.860(3)$ \\
\hline$c / \AA$ & $38.082(9)$ \\
\hline$\beta /{ }^{\circ}$ & $90.588(19)$ \\
\hline Volume $/ \AA^{3}$ & $9979(4)$ \\
\hline $\mathrm{Z}$ & 4 \\
\hline$\rho_{\text {calc }} g / \mathrm{cm}^{3}$ & 2.269 \\
\hline$\mu / \mathrm{mm}^{-1}$ & 9.079 \\
\hline $\mathrm{F}(000)$ & 6232.0 \\
\hline Crystal size $/ \mathrm{mm}^{3}$ & $0.017 \times 0.0029 \times 0.0026$ \\
\hline Absorption correction $\left(\mathrm{T}_{\min } / \mathrm{T}_{\max }\right)$ & numerical (0.1976/0.2998) \\
\hline $2 \Theta$ range for data collection $/{ }^{\circ}$ & 3.612 to 49.998 \\
\hline Index ranges & $-16 \leq \mathrm{h} \leq 16,-20 \leq \mathrm{k} \leq 22,-45 \leq 1 \leq 4$ \\
\hline Reflections collected & 46220 \\
\hline Independent reflections & $17133\left[\mathrm{R}_{\mathrm{int}}=0.0535, \mathrm{R}_{\text {sigma }}=0.0483\right]$ \\
\hline Data/restraints/parameters & $17133 / 0 / 966$ \\
\hline Goodness-of-fit on $\mathrm{F}^{2}$ & 0.965 \\
\hline Final $\mathrm{R}$ indexes $[\mathrm{I}>=2 \sigma(\mathrm{I})]$ & $\mathrm{R}_{1}=0.0291, \mathrm{wR}_{2}=0.0611$ \\
\hline Final $\mathrm{R}$ indexes [all data] & $\mathrm{R}_{1}=0.0440, \mathrm{wR}_{2}=0.0647$ \\
\hline Largest diff. peak/hole / e $\AA^{-3}$ & $2.16 /-1.62$ \\
\hline
\end{tabular}

Details of crystal structure refinement: All non-hydrogen atoms were refined anisotropically. Hydrogen atoms were assigned to idealized geometric positions and included in structure factors calculations. 


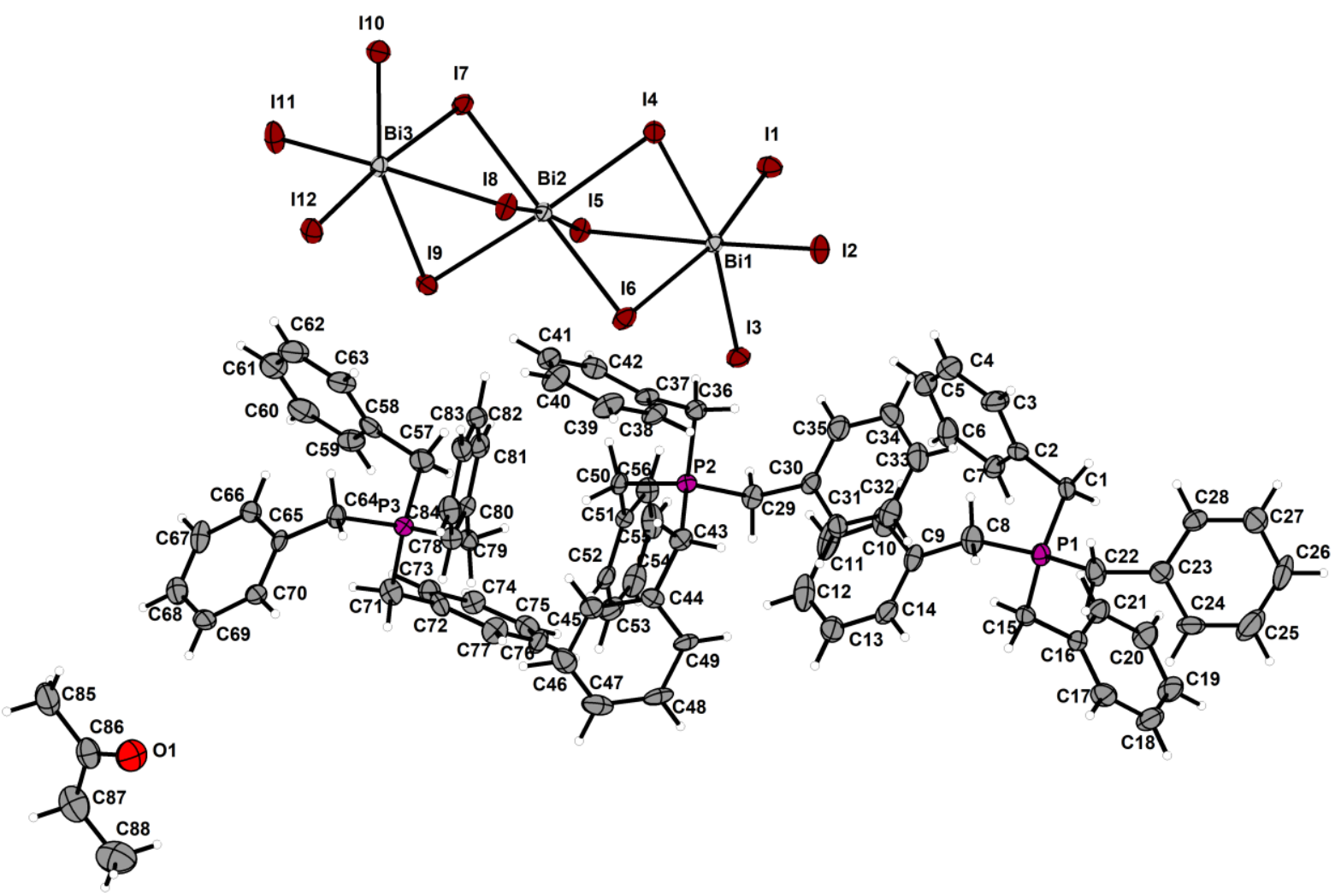

Figure S6: Asymmetric unit of 6, ellipsoids at 50\% probability. 


\section{Powder Diffraction}

Powder patterns were recorded on a STADI MP (STOE Darmstadt) powder diffractometer, with $\mathrm{CuK}_{\alpha 1}$ radiation with $\lambda=1.54056 \AA$ at room temperature in transmission mode. The patterns confirm the presence of the respective phase determined by SCXRD measurements and the absence of any major crystalline by-products unless otherwise indicated.

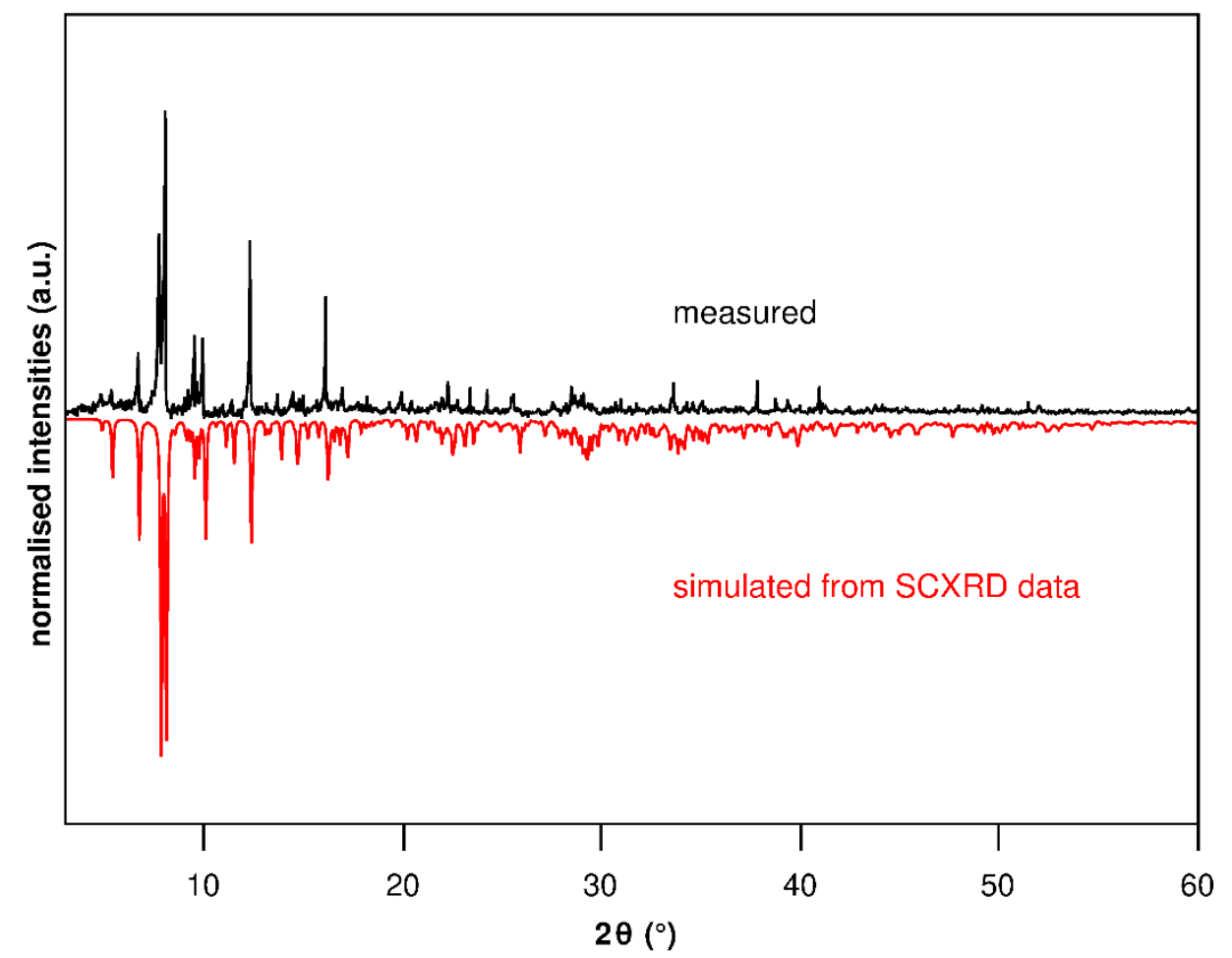

Figure S7. Powder diffraction pattern of $\left(\mathrm{PBz}_{4}\right)_{3} \mathrm{Bi}_{3} \mathrm{Br}_{12}(\mathbf{1})$. 


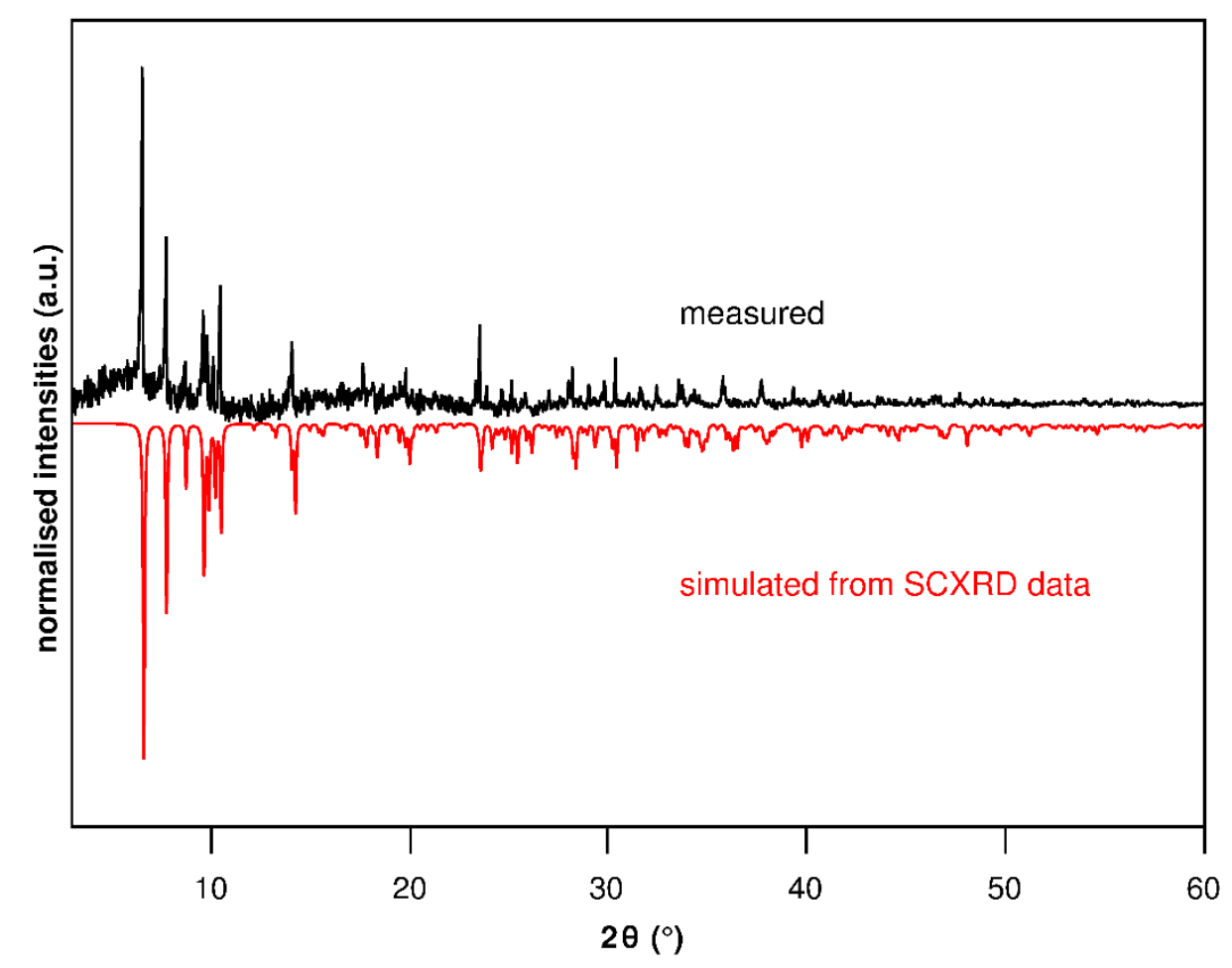

Figure S8. Powder diffraction pattern of $\left(\mathrm{PBz}_{4}\right)_{2}(\mathrm{MeCN})_{2} \mathrm{Cu}_{2} \mathrm{Bi}_{2} \mathrm{Br}_{10}(2)$, prepared from single crystals.

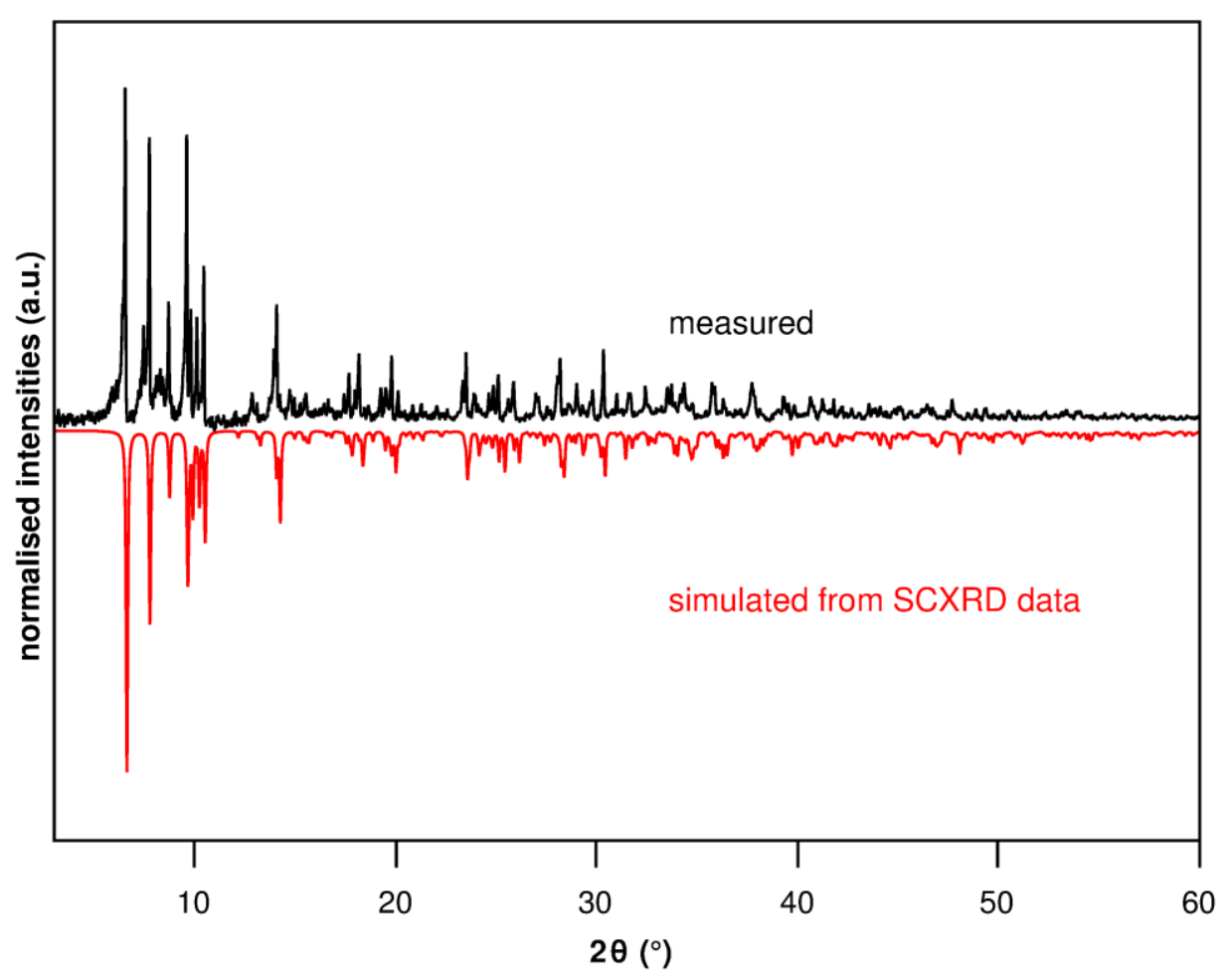

Figure 59. Powder diffraction pattern of microcrystalline powders of $\mathbf{2}$ obtained from direct synthesis showing additional reflections of a small amount of a side phase below $10^{\circ}$. 


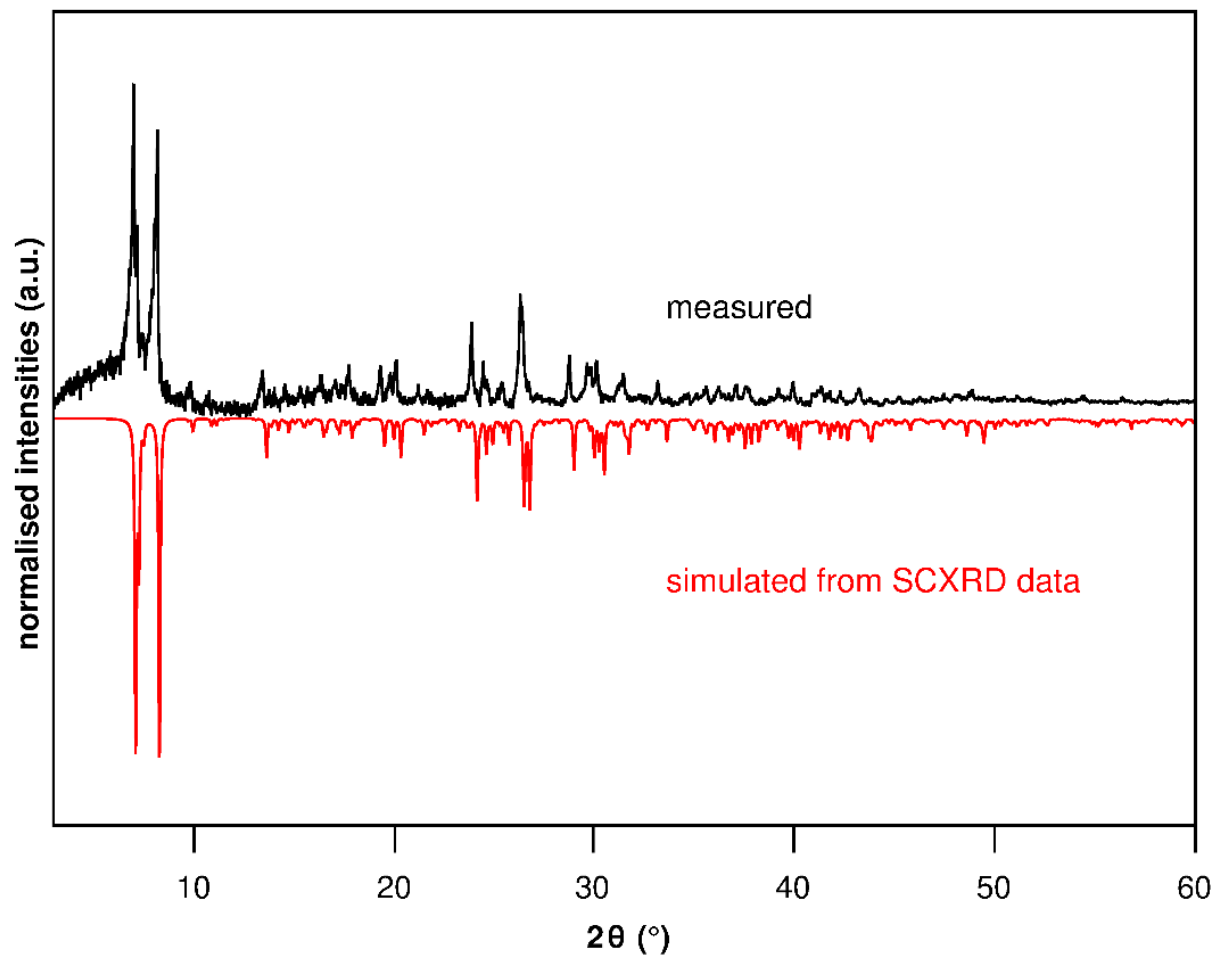

Figure S10. Powder diffraction pattern of $\left(\mathrm{PBz}_{4}\right) \mathrm{Bi}_{2} \mathrm{I}_{7}(\mathbf{3})$. 


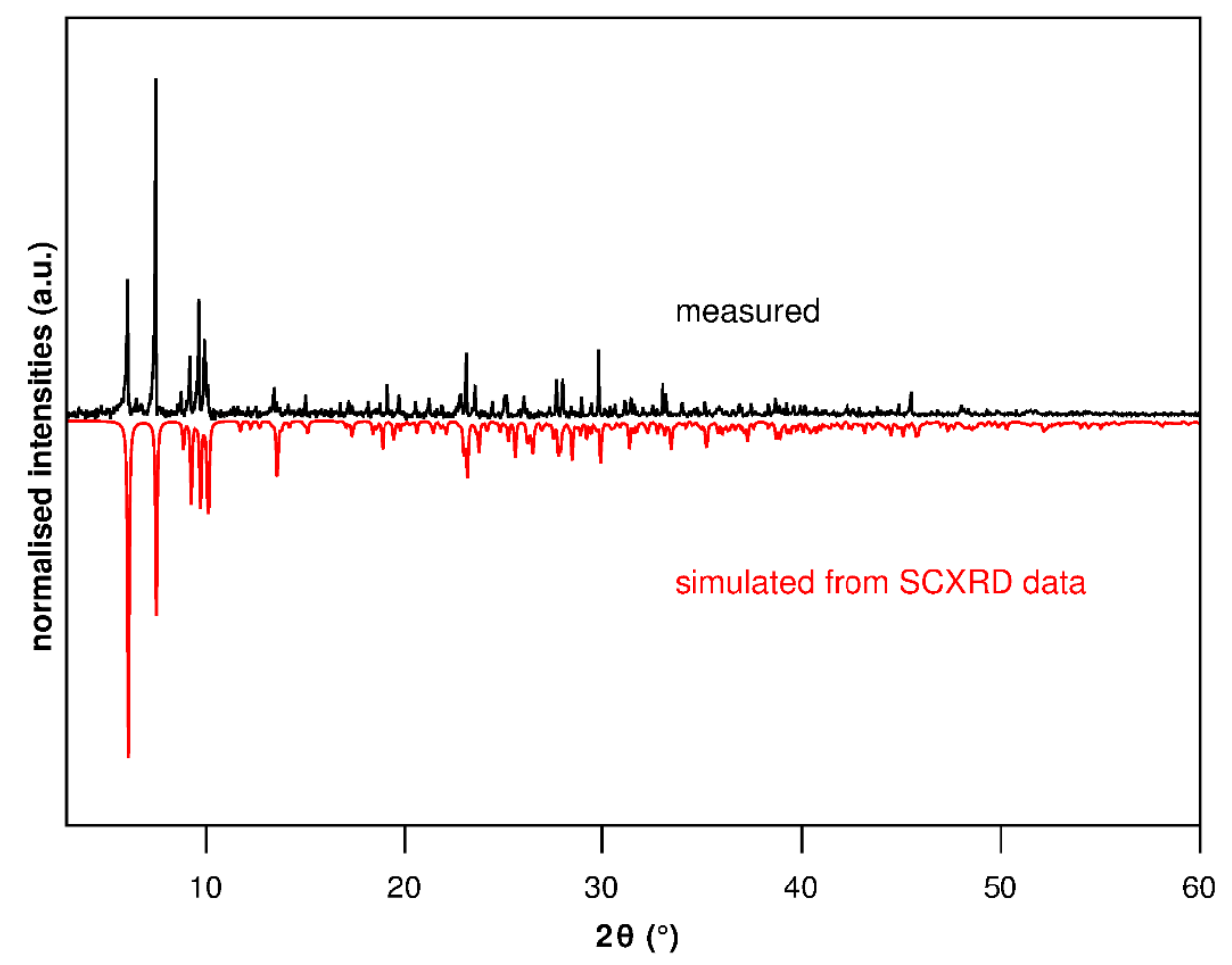

Figure S11. Powder diffraction pattern of $\left(\mathrm{PBz}_{4}\right)_{2}(\mathrm{MeCN})_{2} \mathrm{Cu}_{2} \mathrm{Bi}_{2} \mathrm{I}_{10}$ (4).

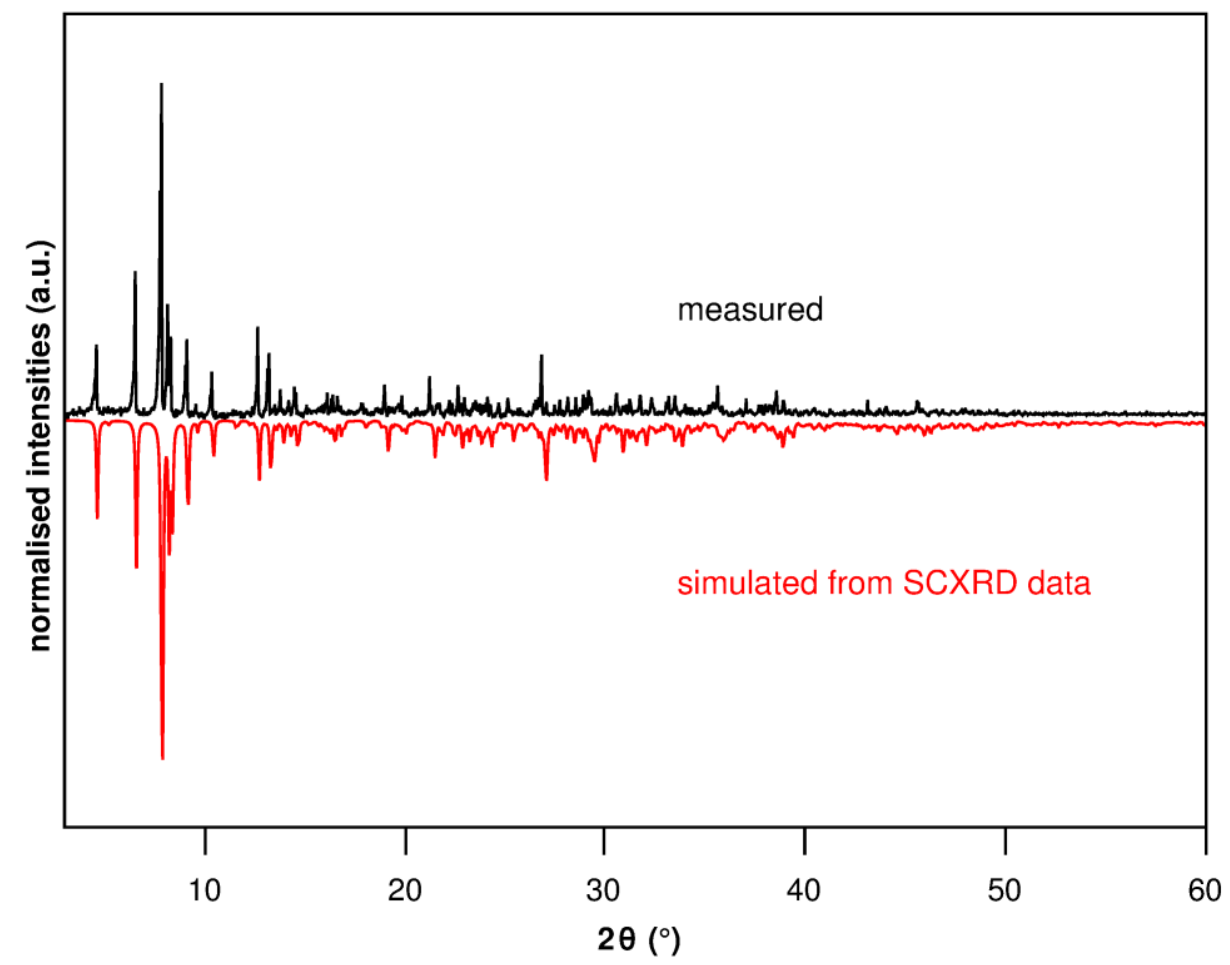

Figure S12. Powder diffraction pattern of $\left(\mathrm{PBz}_{4}\right)_{3} \mathrm{Bi}_{3} \mathrm{l}_{12} \cdot\left(\mathrm{C}_{4} \mathrm{H}_{8} \mathrm{O}\right)(6)$. 


\section{IR spectroscopy}

IR spectra were recorded on a Bruker Tensor 37 FT-IR spectrometer equipped with an ATRPlatinum measuring unit. All spectra show the characteristic bands of the tetrabenzylphosphonium cation. In Figure S17 the IR-spectrum of $\mathrm{PBz}_{4} \mathrm{Br}$ is shown as a reference. In some cases, the characteristic bands of $\mathrm{H}_{2} \mathrm{O}\left(3200 \mathrm{~cm}^{-1}\right)$ and $\mathrm{CO}_{2}\left(2350 \mathrm{~cm}^{-1}\right)$ are visible. Additionally, the band of the $\mathrm{CN}$ group $\left(2270-2400 \mathrm{~cm}^{-1}\right)$ is visible with variable intensity in the spectra of $\mathbf{2}$ and $\mathbf{4}$ and the band of the CO group $\left(1710 \mathrm{~cm}^{-1}\right)$ is present in the spectrum of 6 .

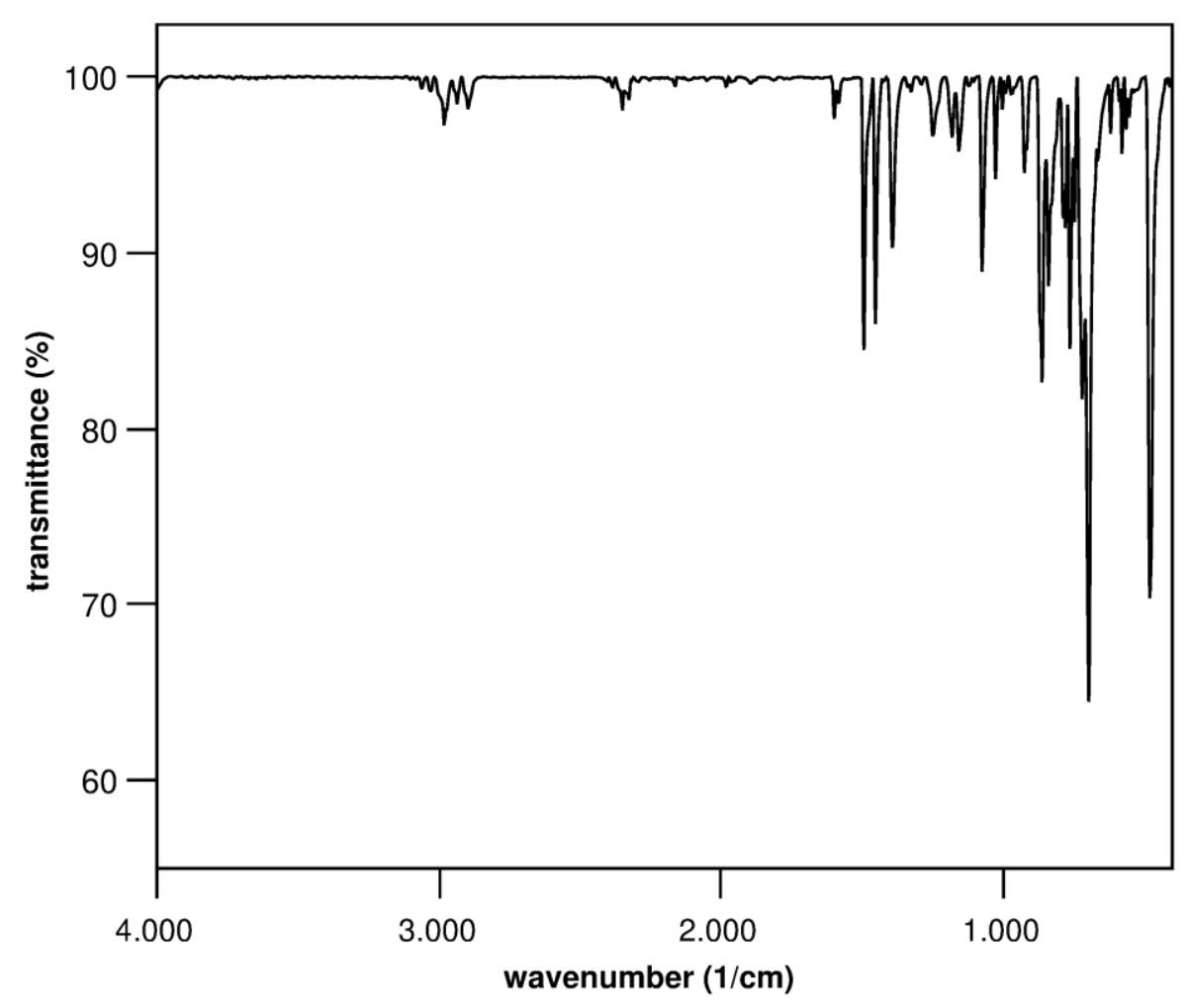

Figure S13. IR spectrum of $\left(\mathrm{PBz}_{4}\right)_{3} \mathrm{Bi}_{3} \mathrm{Br}_{12}(\mathbf{1})$. 


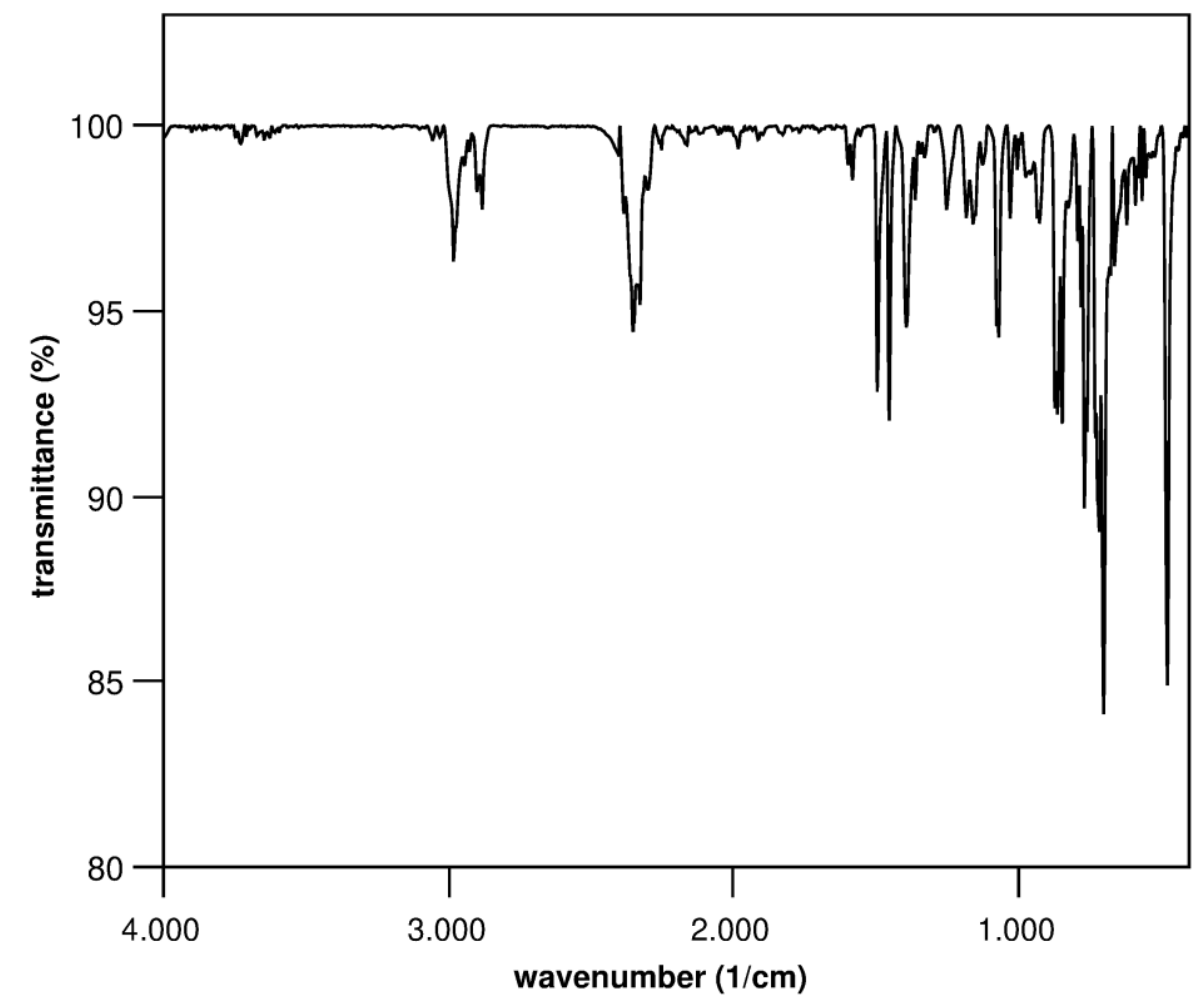

Figure S14. IR spectrum of $\left(\mathrm{PBz}_{4}\right)_{2}(\mathrm{MeCN})_{2} \mathrm{Cu}_{2} \mathrm{Bi}_{2} \mathrm{Br}_{10}$ (2).

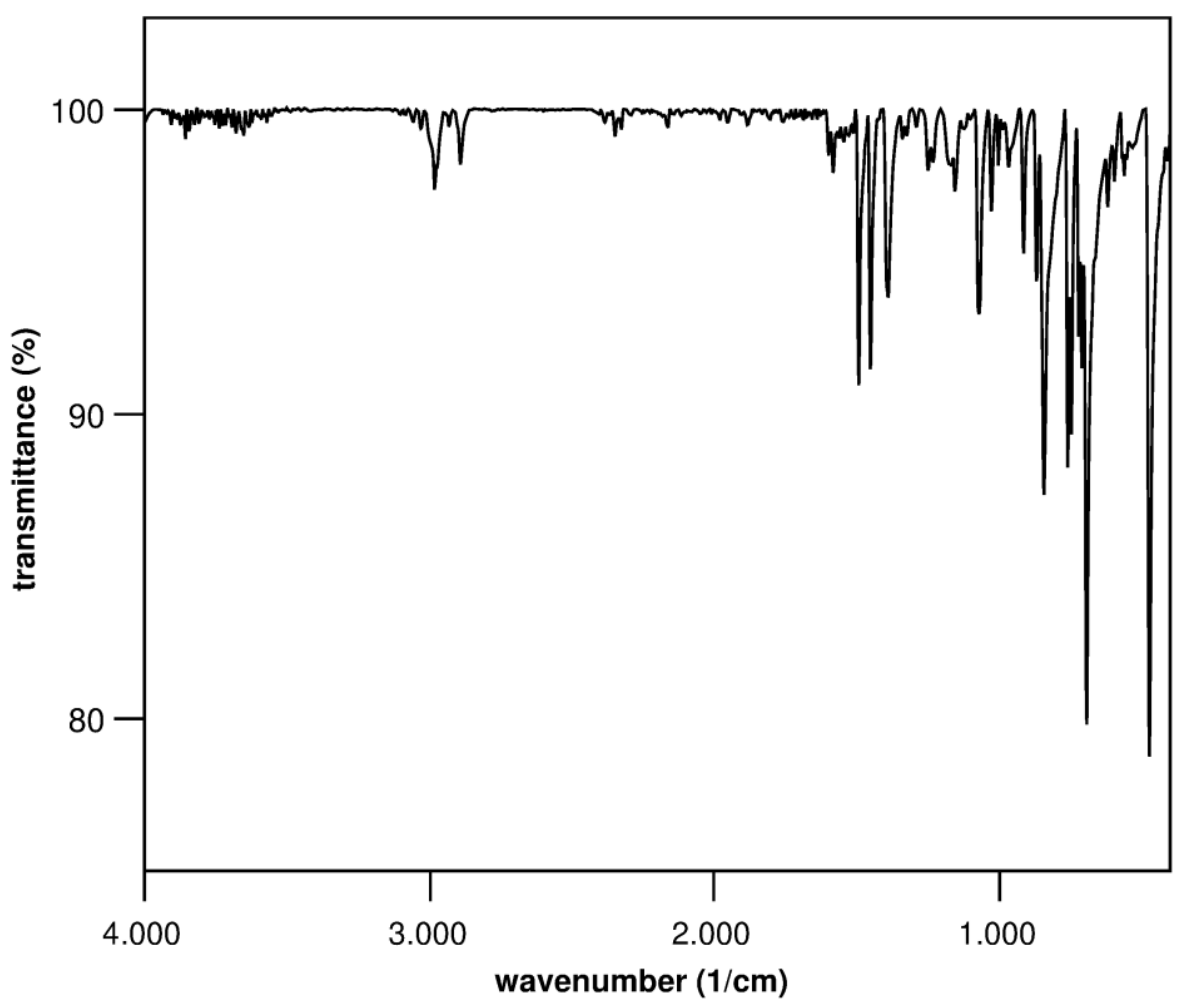

Figure S15. IR spectrum of $\left(\mathrm{PBz}_{4}\right) \mathrm{Bi}_{2} \mathrm{I}_{7}(3)$. 


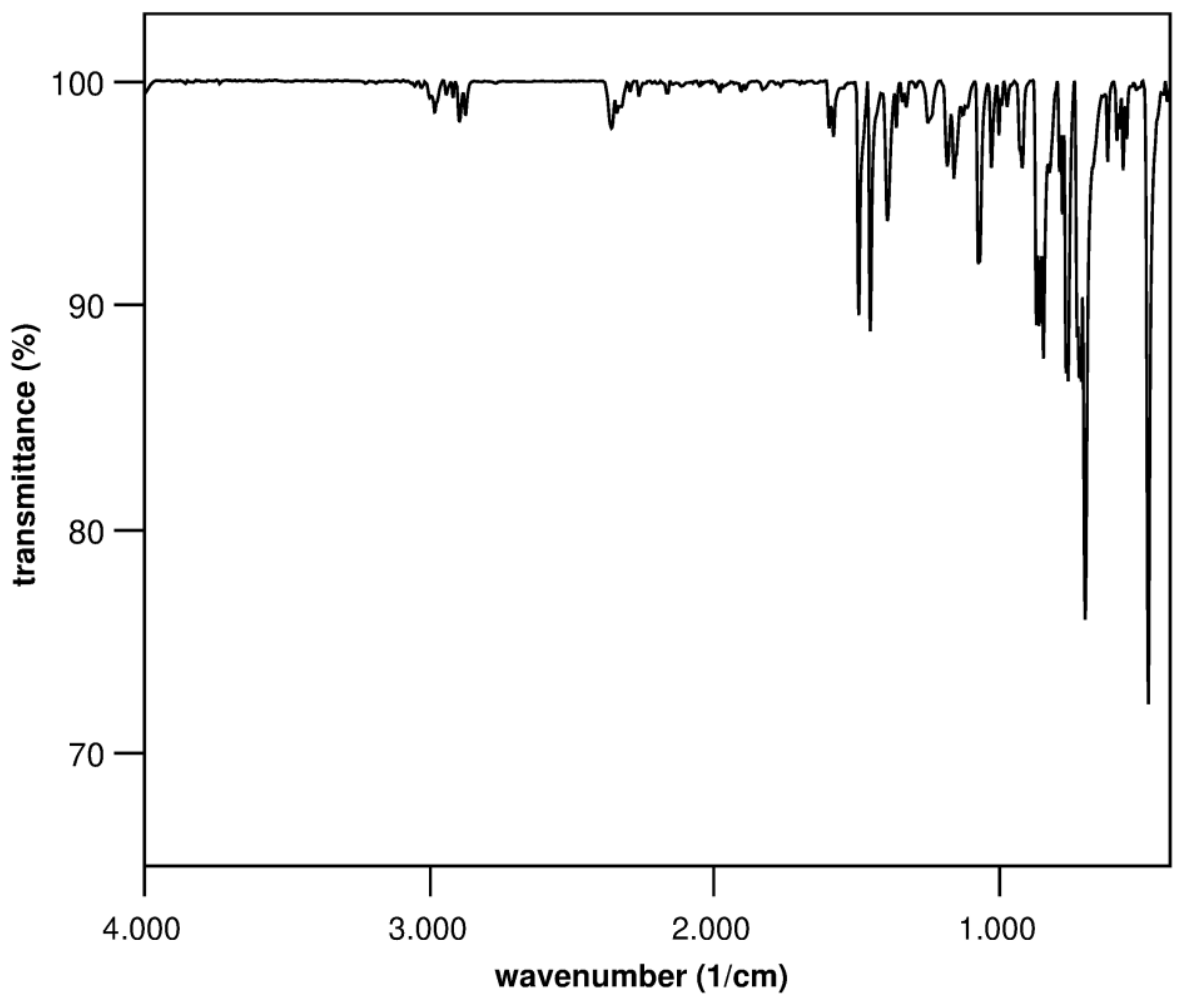

Figure S16. IR spectrum of $\left(\mathrm{PBz}_{4}\right)_{2}(\mathrm{MeCN})_{2} \mathrm{Cu}_{2} \mathrm{Bi}_{2} \mathrm{l}_{10}$ (4).

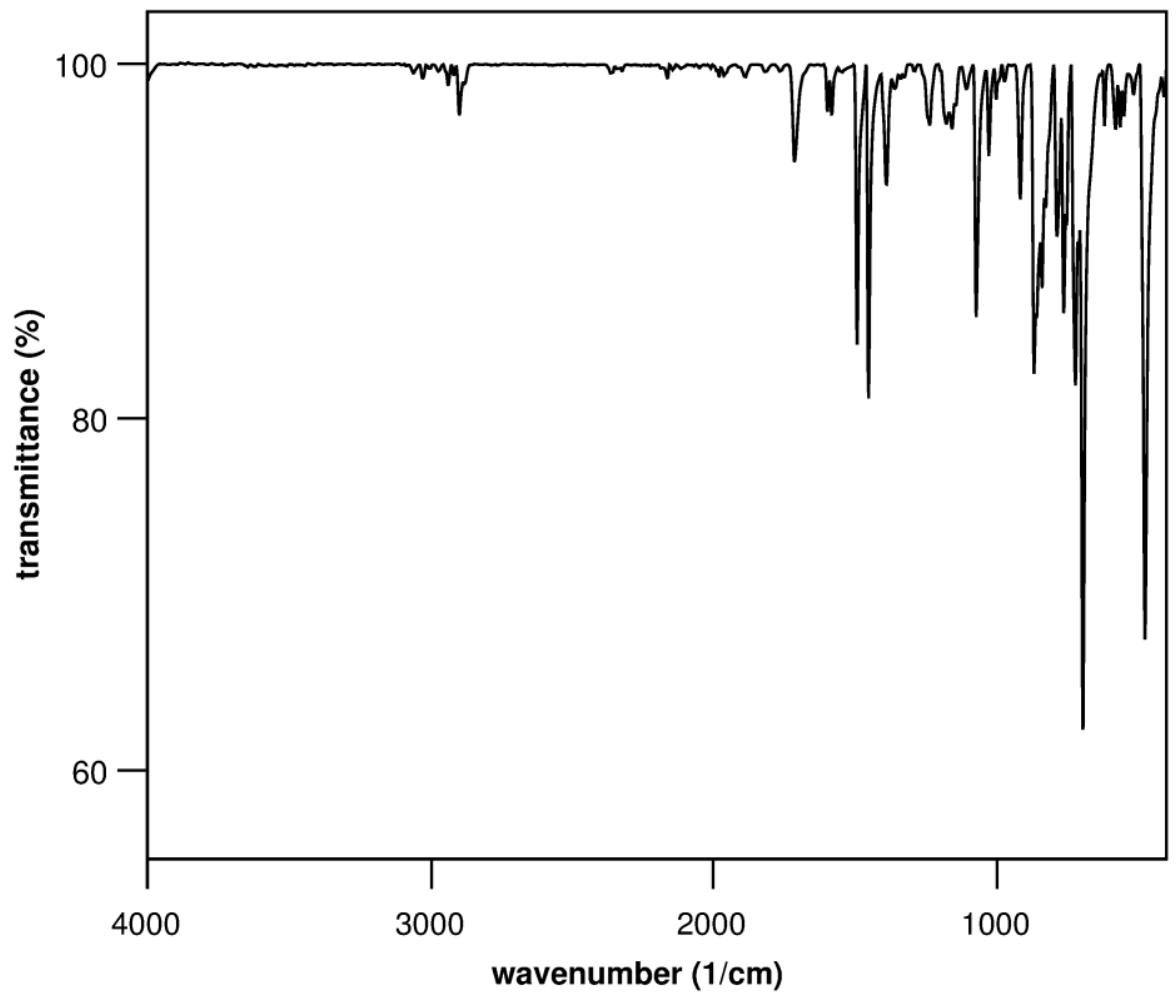

Figure S17. IR spectrum of $\left(\mathrm{PBz}_{4}\right)_{3} \mathrm{Bi}_{3} \mathrm{I}_{12} \cdot\left(\mathrm{C}_{4} \mathrm{H}_{8} \mathrm{O}\right)(6)$. 


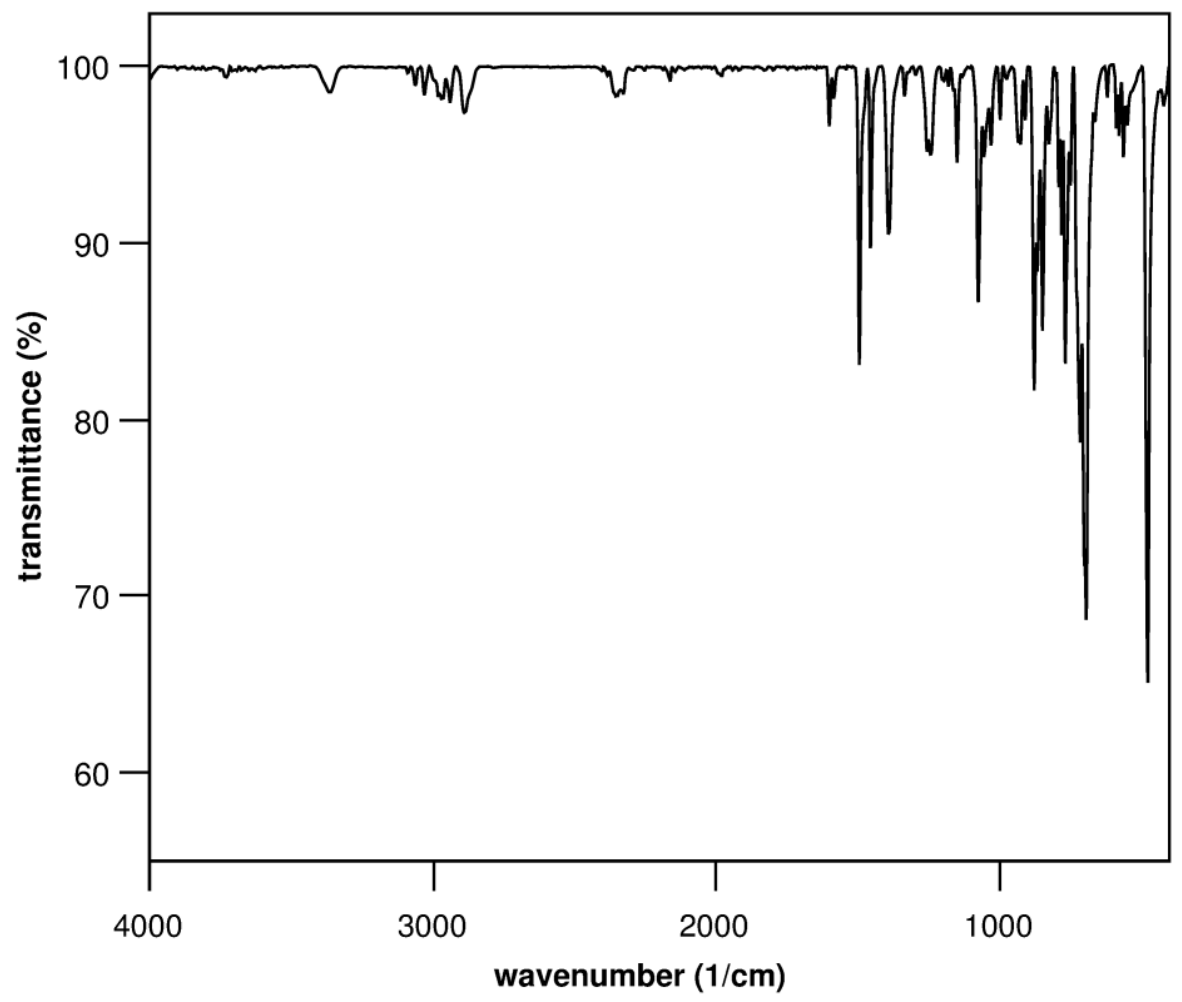

Figure S18. IR spectrum of $\left(\mathrm{PBz}_{4}\right) \mathrm{Br}$. 